\title{
El desarrollo de la escritura en situaciones de contacto lingüístico: un estudio de caso* $^{*}$
}

\author{
The development of writing in linguistic contact situations: a \\ case study
}

Nicole Riveros Diegues**

\begin{abstract}
RESUMEN
En contextos escolares caracterizados por la diversidad cultural, conviven representaciones sociales y usos lingüísticos diversos. Estas construcciones posibilitan la concreción de identidades con las que nos situamos en el mundo y a través de las cuales establecemos relaciones con los otros. En este sentido, el sistema escolar, como uno de los principales medios de socialización, debe garantizar que las habilidades comunicativas promovidas en las prácticas pedagógicas sostengan y refuercen los vínculos entre la lengua y la cultura, sobre todo en aulas interculturales. Por lo anterior, analizamos 31 textos narrativos de mediana extensión y en ellos pudimos evidenciar usos lingüísticos del quechua en construcciones escritas en lengua española. Sostenemos que estos grados de interferencia generan dificultades en la comprensión y producción de los discursos, por lo que sugerimos estrategias didácticas que faciliten el abordaje de la composición escrita en contextos de diversidad lingüística.
\end{abstract}

\section{SUMMARY}

Diverse social representations and linguistic uses coexist in school contexts with cultural diversity. These constructions enable the building of identities in which we place ourselves in the world and through which we establish relationships with others. The school system, as one of the main means of socialization, must guarantee that the communication skills promoted in pedagogical practices sustain and reinforce the links between
Palabras clave: composición, diversidad, habilidades comunicativas, estrategias didácticas, interculturalidad.

Keywords: composition, diversity, communication skills, teaching strategies, interculturality.

\footnotetext{
* Proyecto ANT 1855 Convenio Marco Universidad de Antofagasta

** Chilena. Doctora en Filología española, Universidad Autónoma de Barcelona. Académica de la Universidad de Antofagasta, Antofagasta, Chile. Nicole.riveros@uantof.cl
} 
El desarrollo de la escritura en situaciones de contacto lingüístico: un estudio de caso | Riveros

language and culture, especially in intercultural classrooms. For that reason, we analyzed 31 narrative texts of medium length and in them we were able to witness linguistic uses of Quechua in constructions written in the Spanish language. We argue that these degrees of interference generate difficulties in the understanding and production of speech, so we suggest didactic strategies that facilitate the approach to written composition in contexts of linguistic diversity. 


\section{Desarrollo argumentativo}

Las sociedades siempre se han caracterizado por presentar flujos migratorios, relaciones sociales bidireccionales y confluencia de culturas. Estos fenómenos se han acrecentado en la actualidad, debido a factores sociales, culturales, políticos y económicos. Bajo el propósito de búsqueda de progreso, la mayoría de los sujetos se moviliza para su logro; y la escuela, como uno de los principales motores de socialización y culturización, ha sido testigo de cómo las sociedades mutan y conviven en diversidad.

Se ha estudiado extensamente el rol del sistema escolar en contextos interculturales y se ha concluido que, en entornos de variación cultural, este sistema juega un rol fundamental en la inclusión lingüística y social de los hablantes, lo que no solo repercute en el desarrollo de climas pedagógicos más o menos favorables, sino también en el progreso de las prácticas académicas de los estudiantes.

A su vez, las habilidades comunicativas son imprescindibles para favorecer las relaciones interpersonales que establecen los escolares extranjeros con sus pares, con sus profesores y con el entorno en general. De ahí la importancia de focalizar el desarrollo de la comprensión y producción de textos, ya que estos mecanismos de alfabetización funcional constituyen los medios para la inserción social.

Para analizar estos aspectos, investigamos el tratamiento que una escuela fronteriza del norte de Chile le da a la diversidad lingüística y social de sus estudiantes de ascendencia quechua. Profundizamos, además, en el desarrollo de las habilidades de producción (en concreto, de la escritura) y el efecto de este manejo en el alcance de la competencia comunicativa.

El objetivo principal de este trabajo es reflexionar acerca del manejo pedagógico ante la diversidad lingüística y cultural de los escolares, así como comentar, desde el plano gramatical, una selección de textos narrativos escritos por escolares en zona de contacto, de modo de recomendar secuencias didácticas breves que fortalezcan la funcionalidad de los sistemas, sobre todo, el que promueve la escuela (en este caso, el español).

Hemos propuesto estos objetivos, ya que detectamos que la problemática central en el entorno pedagógico en el que intervenimos se asocia con la confluencia de estadios de interferencia lingüística, los 
que repercuten en el desarrollo de habilidades comunicativas de los estudiantes y generan dificultades en el alcance de los propósitos académicos propuestos.

Por lo tanto, desde la mirada lingüística, creemos que el tratamiento de la gramática para reforzar la escritura es un trabajo gradual y sistemático que puede alcanzarse bajo un enfoque didáctico. A su vez, un adecuado manejo de la interculturalidad puede obtenerse si el sistema escolar en su conjunto comprende que, para generar climas pedagógicos positivos, primero se debe trabajar la conciencia social de los profesores desde la Formación Inicial Docente (FID).

\section{Marco teórico}

La Real Academia Española de la lengua (RAE), define dialecto como "variedad de un idioma que no alcanza la categoría social de lengua" (s./f.). Esta variedad está siempre circunscrita a entornos geográficos delimitados, manteniendo la identidad cultural de un pueblo o nación. En este sentido, los dialectos generan mayores lazos de representación e identidad en los colectivos humanos que los idiomas (o lenguas), ya que su conformación lingüística se debe a transiciones culturales que reflejan la idiosincrasia de los pueblos. En otras palabras, a partir de la lengua se perfilan los dialectos, entendidos como variedades lingüísticas asociadas al factor territorial.

Bajo estos lineamientos se comprende que, al existir migración, también habrá convivencia dialectal. Estas representaciones lingüísticas son frecuentemente observables en la sala de clases, pues el sistema escolar es el centro de culturización por excelencia.

Todos estos dialectos, como variedades de lengua, transmiten culturas diversas que deben sintonizarse para generar no solo un clima escolar agradable, sino también un contexto académico que garantice el aprendizaje. Y esta no es tarea sencilla, pues, como ya ha sido mencionado, se requiere que el profesorado cuente con una formación en educación intercultural que le entregue las herramientas necesarias para fomentar disposiciones positivas en los aprendientes.

Hemos sido testigos de cómo la escuela promueve una determinada variedad de lengua, cuestión en la que no estamos completamente en desacuerdo, ya que es importante reconocer que las relaciones aca- 
démicas, sociales y laborales se rigen por códigos determinados que los hablantes hemos de respetar. Estos códigos deben ser enseñados y fomentados en el contexto educativo con una justificación concreta y una posterior reflexión y valoración, sobre todo pragmática, entendiendo que el contexto determina el uso. Sin embargo, con frecuencia observamos dentro de la sala de clases, cómo se jerarquizan las variedades de lengua: una de ellas se considera "prestigiosa" y todas las otras (que son justamente, los sistemas de los alumnos migrantes) quedan rechazadas sin fundamento académico.

Estas son las llamadas variedades periféricas (Andión y Gil, 2013), formas de lengua que el estudiante extranjero reconoce como propias, válidas y a las que, por cierto, atribuye matices afectivos: son los sistemas de sus padres, de sus abuelos, los dialectos en los que construyen sus recuerdos.

Si la segregación dialectal se observa con frecuencia en las salas de clases, preocupa aún más cuando confluyen en ella distintos idiomas. Las lenguas no solo tienen diversos mecanismos de representación, sino medios de selección y combinación que se ajustan a reglas gramaticales particulares. Estas diferencias generan, evidentemente, dificultades escolares, ya que el estudiante debe enfrentarse a la convivencia entre uno y otro sistema, incluso cuando uno de ellos está en proceso de aprendizaje. Es decir, el estudiante debe transitar constantemente (e inclusive, de forma simultánea) entre su lengua materna (L1) y la lengua social que fomenta la escuela (L2), enfrentándose a esta L2 de manera abrupta e impuesta.

De este modo, asumir que la variable dialectal o la lengua de mayor "prestigio" debe constituir el medio de transmisión social (y, por cierto, escolar) sin un fundamento pragmático, es relegar la importancia de las otras manifestaciones dialectales que presentan tanta viveza e identidad cultural como aquella considerada socialmente estándar. Esto hace que, en muchos casos, los estudiantes extranjeros reconozcan su sistema como inferior y lo visualicen como un impedimento para el ascenso y la inserción escolar (buen rendimiento, gusto por las asignaturas, relaciones interpersonales, entre otros aspectos). Es decir, el tratamiento que se les dé en la escuela a las variedades de lengua, puede convertirse en un factor que genere desmotivación por el aprendizaje, e inclusive, provocar el abandono escolar (Corbo, 2007). 
En este estudio nos hemos centrado en la lengua, puesto que esta es el medio de comunicación y representación por excelencia. Nos interesa indagar cómo se aborda su enseñanza y aprendizaje, pero más allá del análisis de su manejo, nos importa generar estrategias y actividades concretas que produzcan un impacto positivo en la inclusión cultural.

De la lengua, nos centramos particularmente en la escritura, ya que en ella los fenómenos gramaticales interferidos se plasman con mayor concreción, sobre todo porque responden a un ejercicio de planificación y revisión del escrito.

Constatamos que los estudiantes redactan desde un sistema interferido tanto por el español (considerada como lengua materna) y el quechua (la lengua de los padres y abuelos, pero no la de los escolares). Como el quechua es una lengua de base oral, en los registros de escritura en español se evidencian hallazgos de oralidad propios de la lengua amerindia. Resulta interesante analizar y trabajar este fenómeno en el sistema escolar para afianzar la competencia comunicativa.

La base de esta discusión se centra en el rol de la escuela en el desarrollo de la composición escrita. Por largos años, este contexto abordó la escritura como producto, llevando su mirada a los aspectos formales de construcción (ortografía, disposición de párrafos y títulos, estructura en general), pero descuidó el fondo: entender que la composición es un proceso de comunicación, una trayectoria en la que el productor (en este caso, el alumno) interactúa constantemente con su escrito. La composición es entonces el resultado de una sumatoria de fases internas: coherencia, cohesión y adecuación, son aspectos de contenido que, para su logro, requieren de un ejercicio de elaboración consciente y revisión continua.

Otro rasgo muy particular en la escuela es relegar la escritura a un ejercicio de reproducción (dictados y copia de pizarrón), lo que inhibe la destreza del alumno en la escritura y no fomenta el desarrollo de estrategias productivas.

Creemos fehacientemente que este debe ser el foco de la escritura, pensada como estrategia, cuyo trabajo deber realizarse de manera sistemática y contextualizada. Los profesores tienden a abordar la composición escrita bajo tareas mecánicas y sin planificación, y los estudiantes asumen este trabajo no competencial como una labor tediosa, centrada 
en la forma y carente de sentido. Por el contrario, si asumimos que la escritura es un ejercicio progresivo, trabajaremos en función de la habilidad y no del producto. Trabajar este enfoque en un aula monolingüe es un desafío, pero lo es aún más en aquella sala que presenta diversidad lingüística. En este tipo de contextos, el desarrollo de esta habilidad debe planificarse y orientarse a la funcionalidad de los sistemas. Visibilizar en el estudiante que la gramática debe conocerse y aplicarse con conocimiento permitirá que sus escritos contengan mayor información y el contenido de su mensaje se ajuste a las intenciones y contextos.

Con todo lo anterior, aquí sugeriremos actividades de reflexión que respondan a una continuidad en el ejercicio de escritura mediante la organización de los factores contextuales (destinatario, situación comunicativa y propósitos discursivos) y la reorganización de los recursos gramaticales que permitirán desarrollar de forma permanente el contenido del escrito. En este sentido, Zayas (2006) enfatiza en la importancia de trabajar la escritura de la reflexión para el logro de la competencia comunicativa. El autor, para desglosar la complejidad de su alcance, engloba en esta dimensión tres subcompetencias: discursiva (usos lingüísticos para el logro de objetivos comunicativos), textual (basado en los principios de cohesión, adecuación y coherencia) y oracional (selección y combinación de palabras para construir oraciones).

Catalá (2014), por su parte, refuerza esta idea y argumenta que la escritura debe ser trabajada como una forma de organización del pensamiento y para ello "requiere de cimientos sólidos, que corresponde a la escuela establecer, con constancia y rigor" (p. 70).

Por todo lo anterior, las discontinuidades en el proceso de composición se traducen a su vez en dificultades en la transición escolar del estudiante, quien asumirá una notoria desventaja en el desarrollo de habilidades lingüísticas superiores, que son evaluadas en pruebas estandarizadas a nivel nacional. Una de estas mediciones es la prueba Simce (Sistema de Medición de la Calidad de la Educación), evaluación que detalla los avances en las habilidades de escritura de estudiantes de $6^{\circ}$ año básico, y evalúa criterios tanto intralingüísticos (intención, destinatario) como extralingüísticos (organización textual, coherencia, cohesión e informatividad).

Así pensada la escritura - como un ejercicio formativo con un fin competencial—, sugerimos las siguientes fases en la producción de un 
escrito, recopilando lo que la trayectoria literaria ya ha definido. Cabe destacar que este proceso no es lineal, sino circular, y en él los estadios internos se entrecruzan:

- Reflexión inicial (considera las preferencias del que construye el texto y los conocimientos que tenga respecto del tema).

- Organización (distribución de párrafos, referentes y grado de información que se transmitirá en el texto).

- Composición reflexiva (selección y combinación de formas, coherencia y cohesión).

- Revisión (adecuación de la gramática y de los principios textuales, ajustes al propósito y al destinatario).

- Reescritura (reelaboración del escrito considerando todas las fases anteriores).

- Reflexión final (valoración del escrito según el contexto comunicativo).

Bajo un modelo circular situado, la composición cobra sentido como una actividad imprescindible para el logro del pensamiento crítico, productivo y transferible de un contexto comunicativo a otro, según las necesidades y propósitos que tenga el constructor del discurso.

\section{Marco metodológico}

Esta investigación se realizó en una localidad fronteriza del norte de Chile, particularmente, en el límite con Bolivia. Se escogió esta zona, ya que por factores geográficos sus habitantes emplean una variedad de español caracterizada por un estilo gramatical fuertemente influenciado por la lengua quechua.

Como la investigación tiene un fin pedagógico, se trabajó con la única escuela de la zona, a la que asistían 31 estudiantes al momento de realizar esta investigación.

Para analizar el estilo gramatical en las composiciones, se prestó atención al trabajo de la escritura, teniendo como referencia los discursos elaborados en español estándar.

Se utilizaron tres métodos para recabar datos:

a) La elaboración individual de un texto narrativo escrito de temática libre, de mediana extensión. 
b) Un formulario escrito para recopilar antecedentes personales de los entrevistados (edad, género y grados de conocimiento de los idiomas).

c) Acompañamiento en aula.

A través de estas estrategias se reconocieron distintos usos gramaticales interferidos, principalmente, aquellos relacionados con la temporalidad discursiva marcada por discontinuidad, e información secundaria no anclada a la principal, es decir, irrupciones entre referente, referencia e información nueva.

Considerando que los 31 informantes (cuyas edades oscilaban entre los 8 y los 14 años) no eran bilingües, sus construcciones forman parte del español andino (variedad del español en zona de contacto), reconocido por la comunidad intervenida como "quechuañol".

\section{Discusión de resultados}

Comentaremos tres fenómenos lingüísticos de la lengua quechua que, al estar fosilizados ${ }^{1}$, fueron distinguidos con mayor frecuencia en las narraciones. Luego, desde el plano discursivo, abordaremos las irrupciones informativas que se distinguen en las progresiones temáticas. El orden de análisis es el que sigue:

a) Omisión de artículos y preposiciones.

b) Precedencia del modificador al núcleo gramatical.

c) Discordancia temporal.

d) Progresión temática.

En cada apartado mostraremos brevemente la presencia de la lengua amerindia en una selección de ejemplos. Para contextualizar cada intervención, entre paréntesis hemos conservado las iniciales y la edad de los informantes.

1 El Diccionario de Términos Clave del Instituto Cervantes, define "fosilización" como un "fenómeno lingüístico que hace que el aprendiente mantenga en su interlengua de manera inconsciente y permanente, rasgos ajenos a la lengua meta relacionados con la gramática, la pronunciación, el léxico, el discurso u otros aspectos comunicativos" (s./f.). 
Primer fenómeno gramatical: omisión de artículos y preposiciones

\begin{tabular}{l} 
Ejemplos \\
\hline a. "era amigo de ( ) puma” (D.C., 11) \\
b. “¿Cuándo vienen ( ) gaviotas?” (J.V., 10) \\
c. "Me gusta ( ) fútbol” (C.S. 9) \\
d. "Donde ellos quedaron ( ) juntarse” (C.C., 14) \\
e. "Encontró manchas ( ) sangre” (A.A., 12) \\
f. "En la frontera ( ) Bolivia” (B.V., 13) \\
g. “( ) Ollagüe hay muchas fiestas" (A.A., 12)
\end{tabular}

En las muestras del primer fenómeno seleccionado, se evidencia la transferencia morfosintáctica de eliminación del artículo como presentador o actualizador de entidades, ya que la lengua quechua no posee esta categoría en su sistema gramatical. Merma (2007) atribuye la ausencia de artículos en el español andino a razones pragmáticas, en el sentido de que el hablante asume que lo esencial es el contenido del nombre y no la capacidad determinadora que entrega el artículo. Por esta razón, se asume que no es un término imprescindible para establecer una relación con el referente que se presenta (ejemplos a, b y c).

Lo anterior se justifica si consideramos que, a diferencia del español, los dialectos del quechua "se apoyan esencialmente en la situación y en las circunstancias en las que se produce el acto verbal" (Merma, 2007, p. 168).

Respecto de las nociones gramaticales que aportan las preposiciones, en el quechua los casos se gramaticalizan como sufijos. En consecuencia, asumen un posicionamiento posnominal contrario al del español. Creemos que esta diferencia justifica la ausencia de estas categorías en los discursos analizados (ejemplos d, e, fy g).

Segundo fenómeno gramatical: precedencia del modificador al núcleo gramatical
Ejemplos
a. “me lo enseñó el idioma quechua la tía” (Y.T., 9)
b. "Un poco sé hablar en quechua" (Y.T., 9)
c. "Estuvo feliz el día en el pueblo" (T.S., 9)
d. "Fueron allá, en la noche fueron" (W.A., 11)
e. "Se escuchó la puerta fuerte" (W.A., 11)
f. "Se encontró en la tierra una papa" (B.C., 11)
g. "La niña asombrada se fue" (B.V., 13)
h. "en ese hermoso tiempo", "al siguiente día” (L.B., 11). 
En esta selección reconocemos alteraciones en las relaciones núcleo-modificador, tanto con base verbal (b, c, d y e), como con base nominal ( $\mathrm{g}$ y h). En relación con la proyección de la red argumental, los ejemplos (a, e y f) posponen el referente que actúa como complemento directo de las formas transitivas "enseñar", "escuchar" y "encontrar", respectivamente. Así, en el ejemplo (a) se antepone la referencia al referente presentando una construcción bajo la distribución Objeto-Verbo-Sujeto (OVS), es decir, las categorías se trasladan y se presentan de forma más flexible que en el español, cuya tendencia responde al orden SVO. A su vez, la construcción prototípica del quechua es anteponer el objeto al verbo.

Es cierto que en la lengua española, alternar la posición del núcleo nominal y del modificador adjetival en el sintagma puede considerarse un recurso lingüístico válido y rentable, sin embargo, se observa que la variedad de contacto del español con el quechua muestra una mayor frecuencia de la anteposición del adjetivo respecto de la que se distingue en el español. Martínez (2004) explica esta rentabilidad comunicativa y señala las diferencias de significado atribuidas a la posición del adjetivo en relación con el nombre: pospuesto al núcleo nominal, entrega mayor aporte semántico, permite inferir objetividad, y asume mayor carácter informativo. A su vez, la anteposición del adjetivo manifiesta "una modalidad menos objetiva y más evaluativa” (p. 9). Estos matices semánticos hacen que la posición de las categorías en un SN no asuma una total flexibilidad.

Tercer fenómeno gramatical: discordancia temporal

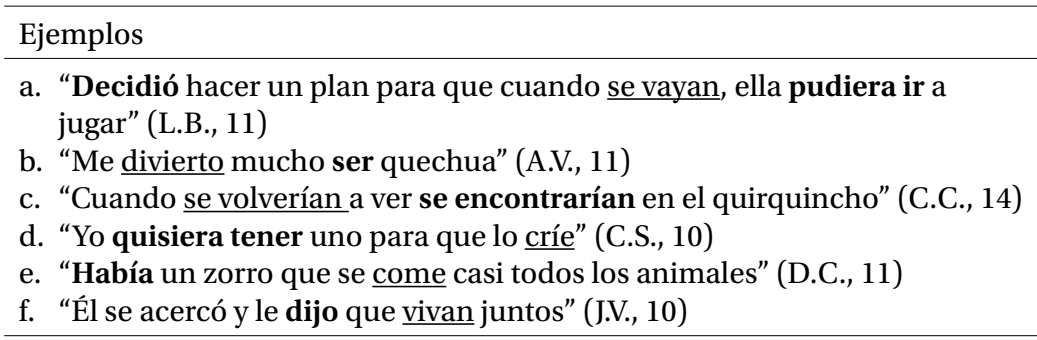

En el tercer fenómeno seleccionado, reconocimos una frecuencia en el uso del pretérito imperfecto y del presente de indicativo, con verbo principal en pasado. Creemos que este tipo de construcciones 
se debe a grados de evidencialidad ${ }^{2}$, rasgo gramatical propio de la lengua quechua. Speranza (2014) señala que probablemente los hablantes de español que se hallan en contacto con lenguas que gramaticalizan la evidencialidad, hacen una explotación de las formas diferente de la que realizan los hablantes monolingües, puesto que el español no posee morfemas con los que gramaticalice dicha función.

Por lo mismo, Gili Gaya (citado en Speranza, 2010) atribuye al modo subjuntivo un carácter de menor certidumbre sobre el contenido referencial de la emisión, expresando acciones en las que el hablante no se compromete con los acontecimientos enunciados por tener un menor grado de certeza. Consideramos que esta es una justificación que se corresponde con los ejemplos que recopilamos, pues las construcciones subordinadas con discordancia temporal expresan estos grados de compromiso evidencial.

\section{Progresión temática}

En el plano de la composición discursiva, comentaremos algunas muestras de escritura de tres estudiantes andinos (informantes A-11, A-23, A-29), quienes a la fecha de recogida de información, tenían 11 años de edad. Como instrucción, se les pidió que escribieran un cuento de extensión media. Para generar representación social con el entorno, el tema del relato tenía que centrarse en la localidad que habitaban. Las trascripciones literales, son las que se presentan:

\section{A-11}

... en las noches salía a buscarlo una noche lo encontró el zorro salto a defenderlo pero el puma empujó a zorro su cabeza coco contra la mesa y fallecio el puma estabal lastimado A medio camino fallecio la chinchilla lloraba y lloraba El zorro todas la noches salía a buscar a su amigo chinchilla.

\section{A-23}

Había una vez un zorro que buscaba agua pasó por amincha no había luego paso por coska tampoco encontró y luego paso por un pubelo que no conocía y ese pueblo se llamaba ollagüe ahí porfin encontró agua tomo agua luego tuvo hambre y se dio una vuelta por ollague y se encontró en la tierra una papa y se la comio y ya no tuvo hambre y luedo se devolvió a amincha y ahí es donde vivio.

2 Speranza (2010) señala que la evidencialidad es "la posibilidad de determinar la evaluación y el grado de compromiso que cada sujeto establece con el contenido referencial transmitido en el enunciado" (p.1). 
A-29

Habia una linda mañana en la escuela y cuando la hora la abian delantado una hora pero por la hora siempre llego tarde toras las tarde le pido para salir ala calle pero siempre ase mucho frio la tarde y temprano atrabes para salir ala calle pero sin frio otro alla en chachaywasi Fin.

Se reconocen en estas muestras hallazgos de oralidad quechua, principalmente por las marcas de evidencialidad registradas y la composición sintáctica propia de la lengua andina. Esta lengua, pese a no ser de competencia de los hablantes, sí se registra en las narraciones y su presencia se debe a una transmisión lingüística transgeneracional.

Sin duda, el desempeño gramatical expresado en las muestras genera conflictos lingüísticos, pero también pedagógicos. Desde el punto de vista de la lengua, se reconoce una notoria dificultad en el aprendizaje de las habilidades comunicativas, discontinuidades discursivas, referentes inconexos y remas sin anclaje. Desde el foco pedagógico, esta interferencia genera inconvenientes en la ejecución de tareas, en la comprensión de los textos y en los ejercicios de producción de los mismos.

Cabe destacar que la fosilización de la interferencia gramatical es aún mayor en la composición escrita, ya que la elaboración de este tipo de discurso (en comparación con la producción oral) responde a un proceso donde la gramática se planifica a conciencia y con mayor determinación.

\section{Sugerencias para abordar la escritura en hablantes bilingües y bidialectales en la escuela intercultural}

Hasta aquí hemos comentado las interferencias gramaticales detectadas en la muestra seleccionada para estos fines. Como estos contactos generan dificultades en el contenido informativo que se transmite, creemos que es de suma importancia proponer actividades que refuercen los usos lingüísticos, de modo que los estudiantes tengan el control de los sistemas que manejan y que, en definitiva, se potencie en ellos la competencia comunicativa.

Enfatizamos en la importancia de esta competencia, pues requiere de habilidades superiores para su logro tales como la reflexión, el análisis y la valoración. Además, es el enfoque académico que se ha 
propuesto en el currículo educativo nacional, cuya concreción se evidencia en los objetivos de aprendizaje dispuestos para ello.

Como la gramática del "quechuañol" se halla fosilizada en los estudiantes, los desajustes lingüísticos se mantienen invisibilizados para ellos, por lo mismo, trabajar la escritura comunicativa no es tarea sencilla y supone un gran desafío. Las estrategias didácticas que proponemos deben desarrollarse de forma paulatina y sistemática, con objetivos definidos y contextualizados, sin descuidar la reflexión y la valoración de las tareas solicitadas. De esta manera, el estudiante se apropiará de su proceso de composición y pasará de la escritura con foco en la forma, a la escritura con foco funcional: aquellos usos inicialmente fosilizados, comenzarán a distinguirse como recursos propios de cada sistema.

\section{Primera sugerencia: la composición sobre la base de modelos discursivos}

Proponemos que la escritura en contextos pedagógicos que presentan casos de interferencia lingüística como los aquí declarados, requiere de un trabajo basado en modelos de escritura estándar, tanto en una lengua como en la otra (en nuestro caso, quechua y español). Por ejemplo, recortes de noticias, cuentos de tradición literaria y cartas, son modelos de textos que enriquecen el proceso, ya sea por la diversidad de formatos que presentan, por la calidad del contenido informativo que transmiten, o bien, por la expresividad de los recursos gramaticales que los conforman. Recomendamos trabajar con textos de referencia en cada lengua, de modo que se refuerce los sistemas según los principios gramaticales que las rigen.

Sobre la base de estos modelos, el docente debe focalizar la atención en los aspectos morfosintácticos que desea que reconozca el estudiante. Por ejemplo, de acuerdo con los tres aspectos comentados en este artículo, el profesor puede desglosar los textos escritos en español y trabajar en grupos, solicitando que distingan núcleos gramaticales y la presencia de artículos y modificadores, comentando cuándo se establece un orden fijo de combinación, y cuándo este orden genera diferencias de sentido.

El estudiante no solo comprenderá que los textos se rigen por soportes informativos distintos y por la información que contienen, sino 
también que responden a estilos de construcción de acuerdo con una normativa gramatical definida para cada sistema lingüístico. Sentirá la necesidad de redactar, a partir de estos modelos discursivos, sus propias composiciones atendiendo a criterios estandarizados.

\section{Segunda sugerencia: análisis comparativo de textos}

En esta segunda actividad didáctica, sugerimos trabajar con los propios textos de los estudiantes, considerando además un texto estándar de referencia escrito en lengua española. Lo importante es que los textos compartan una tipología determinada, es decir, que ambos mantengan aspectos de formato, estilo y contenido. Esta actividad nos permitirá reforzar los usos lingüísticos en el sistema que promueve la escuela como "lengua social".

Así, si queremos atender a la temporalidad discursiva, convendría solicitarle al estudiante la redacción de un texto narrativo. Cuando ya lo haya elaborado bajo un modelo circular y reflexivo, podremos trabajar con un texto que presente similitudes en los aspectos definidos y solicitarle que distinga semejanzas y diferencias entre uno y otro.

Esta comparación dirigida ayudará a que el estudiante comprenda que los textos responden a formas discursivas predeterminadas y que la gramática juega un rol fundamental en cada uno de ellos, por lo que un uso adecuado de las formas lingüísticas contribuirá a la comprensión de sus naturalezas.

\section{Tercera sugerencia: redacción bajo situaciones creadas}

La tercera actividad didáctica que proponemos se basa en una situación creada por el docente de acuerdo con las preferencias de sus estudiantes. Trabajar con la imaginería estimula la producción y hace que el proceso sea más estimulante y significativo.

Proponemos que el docente simule situaciones comunicativas asociadas a espacios geográficos diversos, que le permitan al estudiante enfrentarse con otros usuarios de la lengua sin importar las distancias lingüísticas, sociales y culturales existentes. Esta actividad le ayudará a tomar conciencia de que la variable que utiliza en sus propias composiciones debe ser comprendida en otras zonas geográficas en las que se comparta su lengua, por lo que deberá procurar entregar su texto 
con claridad y precisión, atendiendo a los recursos gramaticales que selecciona y a la forma en que los combina.

Se sugiere que las situaciones creadas por el docente conduzcan a una progresión basada no solo en la diversidad de géneros, sino también en la información contenida. Por último, conviene enfatizar en el rol fundamental que desempeña la retroalimentación en estas propuestas: cualquiera sea la estrategia didáctica seleccionada, la producción escrita debe reforzarse mediante un proceso de reflexión guiado y sistemático.

\section{Conclusiones}

En el análisis aquí expuesto pudimos constatar que los estudiantes que presentan contacto lingüístico quechua-español han adquirido una variedad de español fuertemente interferida por los rasgos de la lengua amerindia. Esta interferencia reconocida como "quechuañol" se refleja en sus escritos y constituye un sistema de interlengua ${ }^{3}$ que debe estabilizarse para evitar irrupciones gramaticales y discontinuidades comunicativas - rasgos como los ya comentados-, entre ellos: la omisión y asignación de preposiciones (generando redes argumentales distintas de las del español estándar) y la subordinación caracterizada por una alteración en el tiempo morfológico, reduciendo notoriamente el sistema temporal del español a los usos de modo indicativo. Se corroboró, además, la tendencia a sustituir las expresiones subordinadas por tiempos independientes, como ya la había manifestado Escobar (2000) respecto del español andino de Perú.

Un aspecto interesante de destacar es la transmisión oral del quechua por ascendencia, ya que los escolares son hablantes monolingües de español, de ahí que los rasgos gramaticales evidenciados se mantengan fosilizados, incluso en la escritura. Se verificó además que esta transferencia no responde a un proceso de conciencia lingüística del alumno, sino que más bien es el resultado de un bilingüismo oral por sumersión, de acuerdo con la definición de Snow, 1999 (cit. en Alar-

3 En la entrada de "interlengua" del Diccionario de Términos Clave del Instituto Cervantes, se reconoce como un "sistema lingüístico del estudiante de una segunda lengua o lengua extranjera en cada uno de los estadios sucesivos de adquisición por los que pasa en su proceso de aprendizaje" (s./f.). 
cón, 2002). Esta construcción traspasa los planos discursivos y refleja, en la escritura de los hablantes monolingües, la sintaxis de sus ancestros quechuas.

Si se considera el contexto geográfico de esta investigación (norte de Chile), el español es la lengua social y académica que promueve la escuela. En este sentido, conviene que se fomente en estos estudiantes el trabajo colaborativo y reflexivo de la escritura teniendo como referencia la norma estándar de la lengua española. De esta manera, se garantizará la inclusión a todos los contextos a los que se enfrenten, pues la sociedad se rige por la calidad en el manejo comunicativo que poseen los hablantes y la escuela debe garantizar este manejo para evitar la exclusión.

Entonces, considerando las muestras de contacto lingüístico, se pueden justificar las diferencias gramaticales como fenómenos de reorganización entre los sistemas. Esto da como resultado una morfosintaxis que puede ser interpretada sobre la base de categorizaciones étnicas que demuestran la perspectiva del hablante "como prueba de la influencia de la cultura en la conformación de la gramática" (Martínez, 2015, p. 203).

Por último, si consideramos que la formación en educación intercultural es aún una tarea pendiente, el trabajo en el aula debe abocarse a estrategias didácticas específicas y situadas, como las aquí sugeridas. Creemos que estas secuencias pueden adaptarse a todas las realidades pedagógicas y no necesariamente enfocarse en aspectos lingüísticos. En definitiva, siempre será de gran ayuda contar con secuencias didácticas que fomenten la inclusión en la escuela, sobre todo, en las aulas interculturales.

\section{Referencias bibliográficas}

Alarcón, L. (2002). Bilingüismo y adquisición de segundas lenguas: inmersión, sumersión y enseñanza de lenguas extranjeras. Simposio CONCYTEQ: La investigación y el desarrollo Tecnológico en Querétaro 2002. Santiago de Querétaro, México.

Andión, M. y Gil, M. (5 y 6 de abril de 2013). Las variedades del español como parte de la competencia docente: qué debemos saber $y$ enseñar en ELE/L2. Actas del I Congreso Internacional de 
Didáctica de Español como Lengua Extranjera del Instituto Cervantes de Budapest. Budapest. Hungría.

Catalá, A.V. (2014). Didáctica de planificación en el proceso de escritura. Revista Textos de Didáctica de la Lengua y de la Literatura, (67), 63-72.

Centro Virtual Cervantes (s./f.). Fosilización. En Diccionario de términos clave de ELE. Recuperado el día de mes de año, de https:// cvc.cervantes.es/ensenanza/biblioteca_ele/diccio_ele/diccionario/fosilizacion.htm

Centro Virtual Cervantes (s./f.). Interlengua. En Diccionario de términos clave de ELE. Recuperado el día de mes de año, de https://cvc.cervantes.es/ensenanza/biblioteca_ele/diccio_ ele/diccionario/interlengua.htm

Corbo, E. (2007). Desertores presentes. En cursiva. Revista Temática de Fundación Arcor, 2(2), 5-8.

Escobar, A. (2000). El español en contacto con el quechua en el Perú. Pontificia Universidad Católica del Perú.

Martínez, A. (21 al 23 de noviembre de 2004). Etnopragmática: Una propuesta teórico-metodológica. Ponencia presentada en el Primer Congreso Internacional de Letras, Universidad Nacional de La Plata, Ciudad Autónoma de Buenos Aires, Argentina.

Martínez, A. (2015). ¿Cómo afecta la cultura a la gramática? El caso de los clíticos en el español americano. Revista Círculo de Lingüistica Aplicada a la Comunicación, (61), 186-210. https:// doi.org/10.5209/rev_clac.2015.v61.48472

Merma, G. (2007). Contacto lingüistico entre el español y el quechua: un enfoque cognitivo pragmático de las transferencias morfosintácticas en el español andino peruano [Tesis de doctorado]. https://rua.ua.es/dspace/bitstream/10045/4114/1/ tesis_doctoral_gladys_merma.pdf

Real Academia de la Lengua Española (s./f.) Dialecto. En Diccionario de la Real Academia Española. Recuperado el día de mes de año, de https://dle.rae.es/?id=DeWvE0K

Speranza, A. (2010). "Nos gritaron que nos fuéramos" (¿o que nos vayamos?): El uso variable de los tiempos verbales como estrategia etnopragmática. Ponencia presentada en el IX Congreso Argentino de Hispanistas, La Plata, Argentina. 
Speranza, A. (2014). Evidencialidad en el español americano. La expresión lingüistica de la perspectiva del hablante. Editorial Iberoamericana.

Zayas, F. (2006). La reflexión gramatical en el aprendizaje del uso de la lengua. En M. García (Ed.), La educación lingüistica y literaria en secundaria: materiales para la formación del profesorado (pp. 69-100). Consejería de Educación y Cultura de la Región de Murcia, España. 